\title{
ESTIMATIVAS DE PARÂMETROS GENÉTICOS PARA CARACTERÍSTICAS DE CRESCIMENTO DE CORDEIROS MESTIÇOS SUFFOLK
}

\author{
PRISCILla REgINA TAMIOSO ${ }^{1}$, LAILA TALARICO DiAs ${ }^{2}$, RODRIGO DE ALMEIDA TEIXEIRA ${ }^{2}$, \\ CLÁUdIO JOSÉ ARAÚJO DA SILVA ${ }^{3}$ \\ ${ }^{1}$ Pós Graduanda da Universidade Federal do Paraná, Curitiba, PR, Brasil. priscillatamioso@gmail.com \\ ${ }^{2}$ Professores Doutores da Universidade Federal do Paraná, Curitiba, PR, Brasil. \\ ${ }^{3}$ Pós Doutorando da Universidade Federal do Paraná, Curitiba, PR, Brasil.
}

\begin{abstract}
RESUMO
Objetivou-se estimar os coeficientes de herdabilidade e correlação genética entre os pesos ao nascer $(\mathrm{PN})$ e à desmama (PD) de cordeiros mestiços Suffolk, pertencentes a rebanho experimental. Assim, foram testados quatro modelos, os quais consideraram o efeito genético aditivo direto (Modelo 1), os efeitos genético direto e de ambiente permanente materno (Modelo 2), os efeitos aditivo direto, de ambiente permanente e genético materno $\left(\operatorname{cov}_{\mathrm{a}, \mathrm{m}}=0\right)$ (Modelo 3 ), e no Modelo 4, além daqueles contemplados no Modelo 3, foi considerada $\operatorname{cov}_{\mathrm{a}, \mathrm{m}} \neq 0$. Após comparar os modelos por meio do teste de

razão de verossimilhança (LRT), observou-se que o 3 foi o mais adequado, sendo que as estimativas de herdabilidade foram de baixa magnitude para PN e PD, iguais a 0,08 $\pm 0,009$ e $0,24 \pm 0,17$, respectivamente, indicando pequena possibilidade de resposta à seleção direta. Da mesma maneira, o coeficiente de correlação genética entre estas características foi $0,43 \pm 0,39$, resultado que implica pequena possibilidade de resposta correlacionada. A inclusão dos efeitos maternos é necessária para a estimação adequada de parâmetros genéticos para as características avaliadas.
\end{abstract}

PALAVRAS-CHAVE: comparação de modelos; correlação genética; herdabilidade; peso corporal.

\section{ESTIMATES OF GENETIC PARAMETERS FOR GROWTH TRAITS IN SUFFOLK CROSSBRED LAMBS}

\section{ABSTRACT}

The aim of this study was to estimate the heritability and genetic correlation coefficients between weights at birth (BW) and weaning (WW) in Suffolk crossbred lambs, which belonged to an experimental herd. Thus, four different animal models were fitted, including the direct additive genetic effect (Model 1), the direct additive genetic and maternal permanent environmental effects (Model 2), the direct additive, permanent environmental and maternal genetic effects $\left(\operatorname{cov}_{\mathrm{a}, \mathrm{m}}=0\right)($ Model 3$)$, and in Model 4 , in addition to those in Model 3, $\operatorname{cov}_{\mathrm{a}, \mathrm{m}} \neq 0$ was considered. After comparing the models through the likelihood ratio test (LRT), Model 3 was chosen as the most appropriate, being the heritability estimates low in magnitude for $\mathrm{BW}$ and $\mathrm{WW}$, equal to $0.08 \pm 0.009$ and $0.24 \pm 0.17$, respectively, which indicates low possibility of response to direct selection. Likewise, the correlation coefficient between these traits, $0.43 \pm 0.39$, implies low possibility of correlated response. The inclusion of maternal effects is necessary for the accurate estimation of genetic parameters for the evaluated traits.

KEYWORDS : body weight; genetic correlation; heritability; model comparison. 


\section{INTRODUÇÃO}

Estudos sobre o crescimento de animais de produção são relevantes para programas de melhoramento genético, os quais são elaborados com base no conhecimento dos parâmetros genéticos de características de interesse econômico. Por meio dos coeficientes de herdabilidade e da correlação genética, é possível predizer se determinadas características responderão à seleção direta e, portanto, se a inclusão das mesmas como critérios de seleção possibilitará ganhos genéticos nos rebanhos. Dentre estas, as de crescimento, como pesos ao nascer, ao desmame e em idades subsequentes, podem ser citadas como importantes critérios de seleção em ovinos de corte e, a partir delas, são realizadas as avaliações genéticas.

Estimativas de parâmetros genéticos para pesos corporais de cordeiros em diferentes idades estão amplamente descritas na literatura e apresentam variações de magnitude, dependendo do modelo utilizado. A escolha do modelo adequado na obtenção de parâmetros genéticos pode levar em consideração os efeitos aditivo direto, genético materno e de ambiente permanente materno. Estudos com diferentes raças de ovinos mostram que ambos os efeitos genéticos direto e materno atuam em períodos pré e pós-desmama de cordeiros $^{1-3}$, indicando que, quando não incluídos nos modelos de análise, as estimativas tornam-se inflacionadas.

Entretanto, no Brasil, ainda existem poucas estimativas de parâmetros genéticos para ovinos da raça Suffolk. Assim, o objetivo do presente estudo foi estimar os coeficientes de herdabilidade $\mathrm{e}$ correlação genética entre as características peso ao nascer e ao desmame de cordeiros mestiços Suffolk, a partir de diferentes modelos de análise.

\section{MATERIAL E MÉTODOS}

Foram utilizados os dados históricos do rebanho experimental pertencentes ao arquivo zootécnico do LAPOC - Laboratório de Produção e Pesquisa em Ovinos e Caprinos, da Universidade Federal do Paraná - UFPR, localizado na Estação Experimental do Canguiri, no estado do Paraná, sul do Brasil.

Para as análises, foram formados dois arquivos com dados de peso ao nascer (PN) e peso ao desmame (PD) (realizado entre 45 e 70 dias), de cordeiros mestiços Suffolk, coletados no período de 2002 a 2012. A ausência e/ou a pequena quantidade de informações de pesos corporais além do período da desmama (não incluídas no presente estudo) podem ser justificadas pelo perfil de pesquisa do LAPOC, o qual desenvolve diferentes estudos relacionados à validação de sistemas de produção, sobretudo com animais jovens.

Para PN foram incluídos, como fixos, os efeitos de ano de nascimento, o sexo e a estação de nascimento, classificada em seca (primavera e verão) e chuvosa (outono e inverno) e, como covariáveis, os efeitos linear de tipo de parto, como simples ou múltiplo (duplo ou triplo) e linear e quadrático da idade da ovelha ao parto, em anos. Da mesma maneira, para PD foram considerados como fixos os efeitos de ano de nascimento, o sexo, a estação de nascimento, classificada em seca (primavera e verão) e chuvosa (outono e inverno), e o grupo de manejo ao desmame e, como covariáveis, os efeitos linear de tipo de parto, simples ou múltiplo (duplo ou triplo), linear e quadrático da idade da ovelha ao parto (em anos), além do efeito linear da idade do cordeiro. Animais sem informação de peso e grupo de manejo, bem como ovelhas com idade superior a sete anos foram retirados do arquivo. À edição dos dados, os arquivos de PN e PD contaram com 1.022 e 642 animais, respectivamente. $\mathrm{Na}$ Tabela 1 está apresentada a estatística descritiva para ambos os conjuntos de dados.

Os modelos estatísticos utilizados para estimar os parâmetros genéticos podem ser representados matricialmente da seguinte maneira:

Modelo 1: $\mathrm{Y}=\mathrm{X} \beta+\mathrm{Za}+\mathrm{e}$;

Modelo 2: $\mathrm{Y}=\mathrm{X} \beta+\mathrm{Za}+\mathrm{Wp}+\mathrm{e}$;

Modelo $\quad 3: \quad \mathrm{Y}=\mathrm{X} \beta+\mathrm{Za}+\mathrm{Mm}+\mathrm{Wp}+\mathrm{e}$, assumindo a $\operatorname{Cov}(a, m)=0$;

Modelo $\quad 4: \quad \mathrm{Y}=\mathrm{X} \beta+\mathrm{Za}+\mathrm{Mm}+\mathrm{Wp}+\mathrm{e}$, assumindo a $\operatorname{Cov}(a, m) \neq 0$;

Em que: Y é o vetor das observações; $\beta$, a, $\mathrm{m}$, $\mathrm{p}$ são os vetores dos efeitos fixos, genético aditivo, materno e ambiente permanente, respectivamente; $\mathrm{X}, \mathrm{Z}, \mathrm{M}, \mathrm{W}$ são as matrizes de incidência relacionadas aos respectivos efeitos; e é o vetor dos resíduos aleatórios associados a cada observação.

Pressuposições:

$\mathrm{E}(\mathrm{y})=\mathrm{X} \beta$

$\mathrm{E}(\mathrm{a})=0, \mathrm{E}(\mathrm{m})=0, \mathrm{E}(\mathrm{c})=0$ e $\mathrm{E}(\mathrm{e})=0$.

$$
\operatorname{Var}\left[\begin{array}{l}
a \\
m \\
c \\
e
\end{array}\right]=\left[\begin{array}{cccc}
A \sigma_{a}^{2} & 0 & 0 & 0 \\
0 & A \sigma_{m}^{2} & 0 & 0 \\
0 & 0 & I_{n m} \sigma_{c}^{2} & 0 \\
0 & 0 & 0 & I_{n b} \sigma_{e}^{2}
\end{array}\right]
$$

Em que: $A=$ matriz de parentesco genético aditivo; $I_{n m}$ e $I_{n b}=$ matrizes identidade de dimensão 
número de matrizes/ovelhas $(\mathrm{nm}) \mathrm{e}$ total de observações $(\mathrm{nb}) ; \sigma_{\mathrm{a}}{ }^{2}=$ variância genética aditiva direta; $\sigma_{\mathrm{m}}{ }^{2}=$ variância genética aditiva materna; $\sigma^{2}{ }_{\mathrm{c}}$ $=$ variância de ambiente permanente e $\sigma_{\mathrm{e}}^{2}=$ variância residual.

Os componentes de variância e os parâmetros genéticos foram estimados pelo método de Máxima Verossimilhança Restrita (REML), por meio do programa estatístico Wombat ${ }^{4}$. A escolha do modelo mais adequado para cada peso foi realizada a partir do teste de razão de verossimilhança (Likelihood Ratio Test), o qual compara o aumento da função de verossimilhança (avaliado pela significância do acréscimo de um efeito, quando comparado a um modelo em que não é incluso), a uma distribuição qui-quadrado com " $n$ " graus de liberdade e probabilidade de $5 \%$ de erro $(\mathrm{P}<0,05)^{5}$.

Tabela 1. Estrutura do banco de dados para pesos ao nascimento (PN) e ao desmame (PD) de cordeiros mestiços Suffolk

\begin{tabular}{ccc}
\hline Característica & PN & PD \\
\hline Média $(\mathrm{kg})$ & 4,87 & 19,90 \\
Desvio-padrão $(\mathrm{kg})$ & 1,05 & 5,93 \\
Coeficiente de variação $(\%)$ & 21,56 & 29,80 \\
Número de observações & 1.022 & 642 \\
Número de matrizes & 288 & 244 \\
Número de reprodutores & 27 & 27 \\
\hline
\end{tabular}

\section{RESULTADOS E DISCUSSÃO}

A Tabela 2 ilustra os comparativos entre os quatro diferentes modelos por meio do LRT para estimação dos coeficientes de herdabilidade para os pesos ao nascer e à desmama.

Por meio do teste de razão de verossimilhança (LRT) foi possível notar que o Modelo 3, o qual contemplou o efeito genético materno e a covariância entre os efeitos diretos e maternos igual a zero, foi o mais adequado para estimar as herdabilidades das características estudadas (Tabela 2). Foi possível observar também um aumento no valor da função de verossimilhança, bem como maiores erros-padrão nas estimativas de herdabilidade, quando a covariância entre os efeitos genéticos diretos e maternos foi incluída (Modelo 4), gerando contradições entre os Modelos 3 e 4. Este fato evidencia, portanto, que é possível considerar $\operatorname{cov}_{\mathrm{a}, \mathrm{m}}=0$ para estas características, como proposto por Lôbo et al. ${ }^{6}$, em um trabalho em que os autores sugeriram cautela em análises que consideram como diferentes de zero os valores de covariância entre os efeitos materno e direto, pela possibilidade de que tal covariância seja produto da estrutura do banco de dados.

Tabela 2. Comparação entre os quatro modelos propostos e estimativas de herdabilidade direta $\left(\mathrm{h}_{\mathrm{d}}^{2}\right)$, com respectivos erros-padrão (ep) para pesos ao nascimento (PN) e ao desmame (PD) de cordeiros mestiços Suffolk

\begin{tabular}{cccccc}
\hline Características/Modelos & -2log L & $\begin{array}{c}\text { Comparação entre } \\
\text { modelos }\end{array}$ & LRT & $\mathrm{h}^{2}{ }_{\mathrm{d}} \pm \mathrm{ep}$ \\
\hline \multirow{6}{*}{$\mathrm{PN}$} & 1 & 293,792 & $(2-1)$ & $12,94^{* *}$ & $0,59 \pm 0,09$ \\
& 2 & 280,854 & $(3-2)$ & $4,00^{* *}$ & $0,09 \pm 0,007$ \\
& $\mathbf{3}$ & 284,854 & $(3-1)$ & $8,94^{* *}$ & $0,08 \pm 0,009$ \\
& 4 & 286,854 & $(4-2)$ & $6,00^{* *}$ & $0,08 \pm 0,08$ \\
& 4 & 286,854 & $(4-1)$ & $6,94^{* *}$ & $0,08 \pm 0,08$ \\
\hline & 1 & 916,179 & $(2-1)$ & $8,23^{* *}$ & $0,56 \pm 0,15$ \\
$\mathrm{PD}$ & 2 & 924,413 & $(3-2)$ & $3,12^{* *}$ & $0,26 \pm 0,17$ \\
& $\mathbf{3}$ & 927,529 & $(3-1)$ & $11,35^{* *}$ & $0,24 \pm 0,17$ \\
& 4 & 930,645 & $(4-2)$ & $6,23^{* *}$ & $0,24 \pm 0,25$ \\
& 4 & 930,645 & $(4-1)$ & $14,47^{* *}$ & $0,24 \pm 0,25$ \\
\hline
\end{tabular}

**(P<0,01); -2log L: função de máxima verossimilhança; LRT: teste de razão de verossimilhança. 
Para peso ao nascer, Zamani \& Mohammadi $^{7}$, avaliando ovinos da raça Mehraban, também observaram que o melhor modelo de análise incluiu ambos os efeitos, mas não as covariâncias entre eles, uma vez que, ao considerar $\operatorname{cov}_{\mathrm{a}, \mathrm{m}} \neq 0$, não foi verificado aumento na função de verossimilhança. Da mesma maneira, para estimar a $\mathrm{h}^{2}$ para peso à desmama (aos 42 dias de idade, em média), Ligda et al. ${ }^{8}$ notaram que o modelo mais adequado incluiu os efeitos aditivo direto e genético materno (com $\operatorname{cov}_{\mathrm{a}, \mathrm{m}}=0$ ). Por outro lado, Mandal et al. ${ }^{9}$ reportaram que o modelo mais apropriado para estimar os coeficientes de herdabilidade para os pesos aos 45, 60 e 75 dias de idade de cordeiros Muzaffarnagari contemplou apenas os efeitos genético aditivo direto e de ambiente permanente materno.

A partir da Tabela 2, observa-se que o efeito materno apresentou influência significativa sobre os pesos corporais avaliados, certamente em função das idades em que as características foram mensuradas. Este resultado reforça a importância de se considerarem os efeitos maternos até a desmama e, portanto, ignorar tais efeitos levará a conclusão equivocada sobre a possibilidade de resposta à seleção direta, uma vez que as estimativas tornam-se inflacionadas quando apenas o efeito genético aditivo direto é incluído no modelo de análise , 9-11 $^{\text {. }}$

De acordo com os modelos indicados pelo LRT, as estimativas de herdabilidade obtidas foram

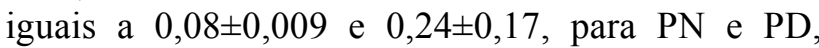
respectivamente, de magnitudes baixa e moderada. Estimativa de herdabilidade baixa $(0,19 \pm 0,04)$ para peso ao nascer de ovinos Suffolk foi encontrada por Maxa et al. ${ }^{12}$, em modelo que considerou os efeitos genéticos aditivo direto e maternos. Da mesma maneira, ainda para esta raça, para peso à desmama (aos 60 dias de idade), Rao e Notter ${ }^{13}$ estimaram herdabilidade direta de baixa magnitude, em análise bivariada, igual a 0,19 , bem como Ekiz et al. ${ }^{14}(0,06)$ e Maroof et al. ${ }^{15}(0,07)$, para as raças Turkish Merino e Avikalin, respectivamente, indicando pequena possibilidade de resposta à seleção direta.

Na Tabela 3 estão apresentados os componentes de (co)variância e as estimativas de parâmetros genéticos a partir dos modelos mais adequados para as características estudadas.

Tabela 3. Componentes de (co)variância e estimativas de herdabilidade e correlação genética entre os pesos ao nascer (PN) e ao desmame (PD), em função do modelo mais adequado (Modelo 3), para cordeiros mestiços Suffolk

\begin{tabular}{cccccccc}
\hline Características & $\sigma_{\mathrm{a}}^{2}$ & $\sigma_{\text {ef }}^{2}$ & $\sigma_{\mathrm{m}}^{2}$ & $\sigma_{\mathrm{p}}^{2}$ & $\mathrm{c}^{2}$ & $\mathrm{~h}_{\mathrm{d}^{2} \mathrm{ep}}$ & $\mathrm{h}_{\mathrm{m}}^{2} \pm \mathrm{ep}$ \\
\hline PN & 0,06 & 0,14 & 0,44 & 0,68 & $0,09 \pm 0,005$ & $0,08 \pm 0,009$ & $0,18 \pm 0,050$ \\
PD & 2,87 & 0,07 & 0,12 & 11,68 & $0,08 \pm 0,006$ & $0,24 \pm 0,170$ & $0,07 \pm 0,040$ \\
\hline
\end{tabular}

$\sigma_{a}^{2}=$ variância genética aditiva direta; $\sigma_{\text {ef }}^{2}=$ variância de efeito de ambiente permanente materno; $\sigma_{m}^{2}=$ variância genética aditiva materna; $\sigma_{p}^{2}=$ variância fenotípica; $c^{2}=$ proporção da variância total devido ao ambiente permanente materno; $h^{2}{ }_{d}=$ herdabilidade direta $; \mathrm{h}_{\mathrm{m}}^{2}=$ herdabilidade materna; $\mathrm{ep}=$ erro-padrão.

Pela Tabela 3, observa-se que a variância genética aditiva direta $\left(h_{d}^{2}\right)$ representou $8-24 \%$ da variação fenotípica total das características avaliadas, com elevados erros-padrão, ao passo que a variância genética aditiva materna representou $18 \%$ e $7 \%$ para PN e PD, respectivamente. Nota-se ainda que os coeficientes de herdabilidade materna estimados para PN e PD apresentaram magnitudes baixas, a partir do modelo mais apropriado. Para $\mathrm{PN}$, o valor encontrado foi semelhante ao reportado por Matika et al. $^{16}(0,12 \pm 0,03)$, e superior aos obtidos por Kushwaha $^{2}(0,09 \pm 0,04)$ e Prince et al. ${ }^{3}(0,08 \pm 0,03)$. Em artigo de revisão, Safari et al. ${ }^{17}$ relataram que as estimativas de herdabilidade materna para peso ao nascer para raças de dupla aptidão (carne e lã) variam entre 0,18 e 0,24 . Já para $\mathrm{PD}$, os coeficientes geralmente apresentam menores magnitudes conforme encontrado em outros trabalhos realizados com diferentes raças de ovinos, como $0,10^{17}, 0,08^{7} \mathrm{e}$ $0,01 \pm 0,01^{3}$, observado também no presente estudo.

A diminuição da importância dos efeitos maternos sobre os pesos dos cordeiros com o aumento da idade do animal é esperada ${ }^{18,}{ }^{19}$, apresentando importância em mamíferos, sobretudo em animais jovens ${ }^{20}$. Outros autores também evidenciam a importância dos componentes maternos e atribuem a maior parte da variação do peso de cordeiros a esses efeitos ${ }^{9,10,21}$, em trabalhos realizados em diferentes períodos de idade, pré e pós-desmama.

As estimativas de correlações genéticas, ambientais e fenotípicas entre PN e PD foram positivas e variaram de magnitudes baixas a altas, porém com altos erros-padrão, indicando pequena 
possibilidade de resposta correlacionada. Acredita-se que a magnitude das estimativas e o elevado erropadrão possam ser justificados pelo número de animais avaliados e pela estrutura da matriz de parentesco deste banco de dados. As características de crescimentos estudadas apresentaram coeficiente de correlação genética entre os efeitos diretos igual a $0,43 \pm 0,39$. Estudos realizados com diferentes raças de ovinos, usando diferentes modelos, mostram que, de maneira geral, pesos ao nascer e à desmama apresentam correlação genética de magnitudes variando de moderadas a altas ${ }^{1,21-25}$. Estes resultados mostram que a seleção para o aumento no peso em determinada idade pode resultar no progresso genético de pesos subsequentes ${ }^{26}$.

Da mesma maneira, a correlação genética entre os efeitos maternos das características estudadas foi positiva e de magnitude moderada, porém com erro-padrão elevado $(0,32 \pm 0,17)$. As correlações genéticas maternas positivas entre PN e pesos posteriores, como PD, indicam que os efeitos maternos sobre os últimos são parcialmente originados do período pré-natal ${ }^{27}$. Já a estimativa de $r_{c}$ entre PN e PD foi positiva e de baixa magnitude, igual $0,15 \pm 0,06$, superior à obtida por Ghafourikesbi e Baneh ${ }^{26}(0,29 \pm 0,27)$, em ovinos Makooei, e inferior às reportadas por Gowane et al. ${ }^{23}$ $(0,79 \pm 0,13)$ e Shokrollahi e Zandieh ${ }^{27}(0,32)$, em trabalhos com ovinos das raças Malpura e Kurdish, respectivamente. Este resultado sugere que as condições de manejo, bem como a relação maternofilial (comportamento materno) e a produção de leite no nascimento e à desmama apresentaram-se como efeitos favoráveis sobre os cordeiros, embora de pequena magnitude.

Similarmente, a correlação fenotípica de magnitude baixa e positiva entre os efeitos $(0,16 \pm 0,09)$ indica presença de relação favorável entre as características de interesse econômico que foram avaliadas. Por fim, a baixa correlação ambiental encontrada no presente estudo $(0,12 \pm 0,09)$ era esperada, evidenciando que os ambientes pré e pós-natal não são relacionados.

\section{CONCLUSÃO}

A importância da inclusão dos efeitos genético materno e de ambiente permanente materno nos modelos para melhor estimar parâmetros genéticos para características de crescimento de ovinos foi evidente.

A seleção para peso à desmama de ovinos de corte pode ser eficiente, visto que o peso tomado nesta idade é um importante critério de seleção para espécies em que o interesse principal é a produção de carne.

\section{AGRADECIMENTOS}

Os autores agradecem ao LAPOC Laboratório de Produção e Pesquisa em Ovinos e Caprinos, da Universidade Federal do Paraná UFPR, pela cessão dos dados, e a CAPES pela concessão de bolsa para o desenvolvimento do estudo.

\section{REFERÊNCIAS}

1. Bahreini Behzadi MR, Shahroudi FE, Vanvleck LD. Estimates of genetic parameters for growth traits in Kermani sheep. J Anim Breed Genet. 2007; 124:296-301.

2. Kushwaha BP, Mandal A, Arora AL, Kumar R, Kumar S, Notter DR. Direct and maternal (co)variance components and heritability estimates for body weights in Chokla sheep. J Anim Breed Genet. 2009; 126:278-287.

3. Prince LLL, Gowane GR, Chopra A, Arora AL. Estimates of (co)variance components and genetic parameters for growth traits of Avikalin sheep. Trop Anim Health Prod. 2010; 42:1093-1101.

4. Meyer K. WOMBAT - A program for mixed model analyses by restricted maximum likelihood. User notes. Animal Genetics and Breeding Unit, Armidale; 2006. 56p.

5. Hogg RV, Craig AT. Introduction to Mathematical Statistics. 5 ed. New Jersey: Prentice Hall; 1995. 564p.

6. Lôbo RNB, Martins Filho R, Penna VM, Lima FAM. Genetic parameters for growth traits of zebu cattle in the semi-arid region of Brazil. Ciênc. Anim. 2000; 10(1):7-12.

7. Zamani P, Mohammadi H. Comparison of different models for estimation of genetic parameters of early growth traits in the Mehraban sheep. J Anim Breed Genet. 2008; 125:29-34.

8. Ligda $\mathrm{CH}$, Gabriilidis $\mathrm{G}$, Papadopoulos TH, Georgoudis A. Investigation of direct and maternal genetic effects on birth and weaning weight of Chios lambs. Livest Prod Sci. 2000; 67:75-80.

9. Mandal A, Neser FWC, Rout PK, Roy R, Notter DR. Estimation of direct and maternal (co)variance components for pre- weaning growth traits in Muzaffarnagari sheep. Livest Sci. 2006a; 99:79-89.

10. Mandal A, Neser FWC, Rout PK, Roy R, Notter DR. Genetic parameters for direct and maternal effects on body weights of Muzaffarnagri sheep. Anim Sci. 2006b; 82:133-140.

11. Ghafouri-Kesbi F, Abbasi MA, Afraz F, Babaei M, Baneh H, Arpanahi RA. Genetic analysis of growth rate and Kleiber ratio in Zandi sheep. Trop Anim Health Prod. 2011; 43:1153-1159.

12. Maxa J, Norberg E, Berg P, Pedersen J. Genetic parameters for growth traits and litter size in Danish Texel, Shropshire, Oxford Down and Suffolk. Small Rumin Res. 2007; 68:312-317. 
13. Rao S, Notter DR. Genetic analysis of litter size in Targhee, Suffolk and Polypay sheep. J Anim Sci. 2000; 78:2113-2120.

14. Ekiz B, Özcan M, Yilmaz A. Estimates of genetic parameters for direct and maternal effects with six different models on birth and weaning weights of Turkish Merino lambs. J. Vet Anim Sci. 2004; 28:383-389.

15. Maroof A, Singh SV, Sushil kumar, Arora AL. Comparison of different methods of heritability estimates for body weights and wool yield traits in Avikalin crossbred sheep. Ind J Small Rum. 2005; 11:121-126.

16. Matika O, Van Wyk JB, Erasmus GJ, Baker RL. Genetic parameter estimates in Sabi sheep. Livest Prod Sci. 2003; 79:17-28.

17. Safari E, Fogarty NM, Gilmour AR. A review of genetic parameter estimates for wool, growth, meat and reproduction traits in sheep. Livest Prod Sci. 2005; 92:271-289.

18. Tosh JJ, Kemp RA. Estimation of variance components of lamb weights in three sheep populations. $\mathrm{J}$ Anim Sci. 1994; 72:1184-1190.

19. Snyman MA, Erasmus GJ, Van Wyk JB, Olivier JJ. Direct and maternal (co)variance components and heritability estimates for body weight at different ages and fleece traits in Afrino sheep. Livest Prod Sci. 1995; 44:229-235

20. Robison OW. The influence of maternal effects on the efficiency of selection: A review. Livest Prod Sci. 1981; 8:121-137.
21. Rashidi A, Mokhtari MS, Safi Jahanshahi A, Mohammad Abadi MR. Genetic parameter estimates of pre-weaning growth traits in Kermani sheep. Small Rumin Res. 2008; 74:165-171.

22. Neser FWC, Erasmus GJ, Van Wyk JB. Genetic parameter estimates for pre-weaning weight traits in Dorper sheep. Small Rumin Res. 2001, 40:197-202.

23. Gowane GR; Chopra A, Prakash V, Arora AL. Estimates of (co)variance components and genetic parameters for body weights and first greasy fleece weight in Malpura sheep. Livest Sci, 2010; 131:94-101.

24. Prakash V, Prince LLL, Gowane GR, Arora AL. The estimation of (co)variance components and genetic parameters for growth traits and Kleiber ratios in Malpura sheep of India. Small Rumin Res. 2012; 108:54-58.

25. Magalhães AFB, Lôbo RNB, Facó O. Estimativas de parâmetros genéticos para características de crescimento em ovinos da raça Somalis Brasileira. Ciênc Rural [Internet]. 2013 [citado 2013 Jun. 05]; 43(5):884-889. Disponível em: http://www.scielo.br/scielo.php?script $=$ sci arttext\&pid $=\mathrm{S}$ 0103-84782013000500021\&lng=pt\&nrm $=$ iso

26. Ghafouri-Kesbi F, Baneh H. Genetic parameters for direct and maternal effects of growth traits of sheep. Archiv Tierzucht. 2012; 55(6):603-611.

27. Shokrollahi B, Zandieh M. Estimation of genetic parameters for body weights of Kurdish sheep in various ages using multivariate animal models. Afr J Biotechnol. 2012; 11(8):2119-2123. 